\title{
Sentiment analysis on IMDB using lexicon and neural networks
}

\author{
Zeeshan Shaukat $^{1}$ (D) Abdul Ahad Zulfiqar ${ }^{1,2} \cdot$ Chuangbai Xiao $^{1} \cdot$ Muhammad Azeem $^{1,3} \cdot$ Tariq Mahmood $^{1}$
}

Received: 19 August 2019 / Accepted: 19 December 2019 / Published online: 2 January 2020

(c) Springer Nature Switzerland AG 2020

\begin{abstract}
To find out what other people think has been an essential part of information-gathering behaviors. And in the case of movies, the movie reviews can provide an intricate insight into the movie and can help decide whether it is worth spending time on. However, with the growing amount of data in reviews, it is quite prudent to automate the process, saving on time. Sentiment analysis is an important field of study in machine learning that focuses on extracting information of subject from the textual reviews. The area of analysis of sentiments is related closely to natural language processing and text mining. It can successfully be used to determine the attitude of the reviewer in regard to various topics or the overall polarity of the review. In the case of movie reviews, along with giving a rating in numeric to a movie, they can enlighten us on the favorableness or the opposite of a movie quantitatively; a collection of those then gives us a comprehensive qualitative insight on different facets of the movie. Opinion mining from movie reviews can be challenging due to the fact that human language is rather complex, leading to situations where a positive word has a negative connotation and vice versa. In this study, the task of opinion mining from movie reviews has been achieved with the use of neural networks trained on the "Movie Review Database" issued by Stanford, in conjunction with two big lists of positive and negative words. The trained network managed to achieve a final accuracy of $91 \%$.
\end{abstract}

Keywords Neural networks · IMDB · Lexicon · Sentimental analysis · Sentimental dictionary

\section{Introduction}

Before the worldwide feast of the Internet, opinions were frequently sought out from acquaintances and friends' circles. Throughout the decision-making process, an important piece of information is the opinions of other people. Nowadays due to the dawn of big data and Internet usage escalation, gathering opinions from masses on a specific subject is possible, which helps to obtain well insights on that subject. With the increasing number of Web sites, blogs, social media platforms, and reviews, there are millions of users who express their sentiments on these platforms and Web sites. The analysis of these opinions and sentiments is necessary and challenging for various evaluation processes. Sentiment analysis is the technique of identifying significant and individual data from a collection of source materials that contain unacknowledged information of people's perspectives [1]. People usually go over blogs, social networking Web sites like Facebook and Twitter and review sites like IMDB and rotten tomatoes to know about the cast and crew of the movie, review, and ratings [2]. To automate the organization and categorization of sentiment from sentiments reviews, sentiment analysis uses text analysis, natural language processing (NLP), and computational techniques [3]. As of today, numerous Web sites permit users to post their opinions which help them to get better insights into their product or services. The aim of sentimental analysis is examining

$\triangle$ Zeeshan Shaukat, zee@emails.bjut.edu.cn; Abdul Ahad Zulfiqar, abdul_ahad241@yahoo.com; Chuangbai Xiao, cbxiao@bjut.edu.cn; Muhammad Azeem, mazeem.qau@hotmail.com; Tariq Mahmood, tmsherazi@ue.edu.pk| ${ }^{1}$ Faculty of Information Technology, Beijing University of Technology, Chaoyang, Beijing 100124, China. ${ }^{2}$ School of Economics \& Management, Beihang University, Haidian, Beijing 100191, China. ${ }^{3}$ Faculty of Computing \& Information Technology, University of Sialkot, Sialkot 51300, Pakistan. 
and determining the attitude of users in regard to various domains, i.e., "negative or positive." The sentiment score and the sentiment polarity are two important issues for sentimental analysis. Sentiment polarity can be represented as a binary value $(1,0)$ which can be negative or positive. It concerns words in the "Bag of Words" model while neglecting the order of words and grammar of the concerned language. A model is known as POS tagging which tags language grammar, particularly (adverbs, verbs, and adjectives). For example, "The book is not good." transforms into determiner (DT)/The, noun (NN)/book, verb (VBZ)/is, adverb (RB)/not, adjective (JJ)/good. The semantic relationship defined above is referred to as the most complex method, and it disregards rational meaning. Sentiment analysis/opinion mining used natural language processing (NLP) to obtain desired information from concerned resources. Sentiment analysis is mostly used for the reviewing of various products in different application domains, for instance market research and customer service, etc. For sentiment categorization, the efficiency of the sentiment lexicons at the sentence level and document level was evaluated using a news headlines dataset and a dataset of Amazon product review [4]. The major purpose of sentimental analysis is to evaluate all opinions to calculate the cumulative polarity of reviews for concerned topics based on levels of classification, for instance negative or positive. Existing concurrences of available reviews could be divided into four levels:

Document level, sentence level, word level, and entity/ aspect level.

\subsection{Applications of sentimental analysis}

Applications of sentimental analysis sneaked into an extensive array of domains, which include financial services, customer services, and products, healthcare, political and social events. Sentimental analysis applications are given a firm ground for research in various industrial interests. For example, in [5] using a sentimental model sales performance was predicted so well. In [6], for categorizing and ranking merchants and product review analyses method was used by the author. In [7], the author relates Twitter sentiments to the opinion of the public on polls. In [8], the author successfully predicted election results using a Twitter sentiment. In [9], a new mechanism was discovered for predicting the volume of comments on political blogs. Nowadays, the Internet turns out to be a major platform for evaluation of public sentiments. The number of active users on the Internet predicted to be 5 billion by 2020 , so there will be huge numbers of reviews produced each day on the Internet worldwide [10]. In [11], a new study label around 90\% of users investigates online about their desired product and their decisions majorly influenced by online reviews about those products. In [12], around $80 \%$ of the client's confidence is totally based on personal review recommendations available online. In this study, we aimed to create a trusted scientific method for evaluation of movie reviews available online. For the purpose of sentimental analysis, we used IMDB extensive in design database of movie reviews issued by Stanford and explored extensively to apply machine learning techniques (Fig. 1).

\subsection{Evaluation of sentimental analysis and its achievements}

The evaluation of sentimental analysis heavily depends on the word level. The structures of reviews are generally short, so the Bag of Words (BoW) mechanism is the most efficient model for analyses purpose. For this study, we used an enhancement BoW mechanism as a baseline to evaluate and analyze sentimental reviews. Enhanced BoW mechanism proposed in this study also carries an easy resolution of challenges usually faced, and the accuracy is improved to a great extent. Each group of available reviews has a different effect on the score of evaluation as it got various features and keywords. The polarity of sentiment can be classified into binary, such as Negative vs. Positive.

Comparative evaluation and system score The comparative evaluation of system score proposed as new criteria for evaluation of reviews. For the measurement of efficiency, a brief comparison is done between the proposed method and other methods based on Accuracy and Performance.

We trained multilayered perceptrons on a database of IMDB movie review by Stanford along with 5000 negative words and 2000 positive words, which gives around $87 \%$ accuracy while testing and around $92 \%$ of accuracy when training.

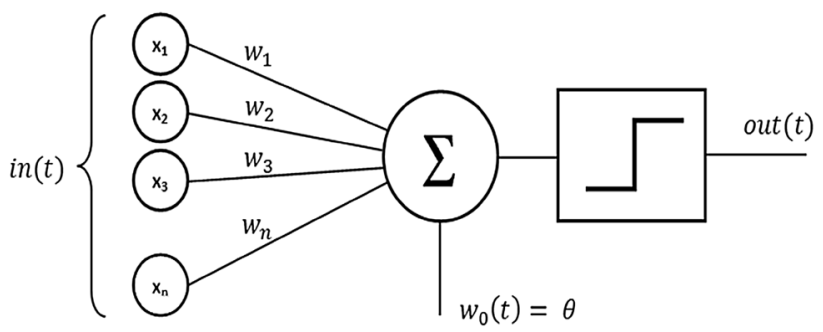

Fig. 1 A simple representation of neural network 


\section{Literature review}

In this section, we will discuss the sentimental analysis and major factors affecting it. Opinion mining also refers to sentiment analysis which is a computational study of reviews, opinions, sentiments, views, and evaluations, etc. The rapid growth of the Internet has produced massive amounts of multilingual information that has been available on different information channels [13]. Nowadays, the scrutiny in evaluating the statistics drawn from social media has been growing because it provides a platform for users to express their views toward things. In this multi-language environment, one challenging but desirable task is to integrate the information in different languages. For this purpose, sentiment detection is used which is an essential process of text analysis in which the primary target is the recognized sentiment within a specific text. Currently, sentiment analysis detection approaches fall under three categories, which are (I) knowledge-based, (II) statistical methods, and (III) hybrid approach. Every method has its strengths and weaknesses [14]. The effectiveness of the sentiment lexicons for sentiment categorization at the document level and sentence level was evaluated using an Amazon product review dataset and a news headlines dataset. Modern and emerging strategies of sentiment detection make use of text illustration nested on semantic vector spaces. Many researchers have used machine learning approaches to detect negation and prediction in sentiment analysis [15]. According to recent reports published in 2019 [13], the quantity of non-English pages is swiftly growing because the growth rate of English Web sites is much lower than many other languages such as Arabic, Chinese, or Spanish. Around 50\% of the Web sites on the Internet are written in non-English languages, and this number is still increasing.

Before we discuss sentimental analysis, we must be aware of the term sentiment. Sentiments can be described as feelings, emotions, opinions, or judgments [16]. In this study, our focus is not on emotions or feelings while the actual emphasis on sentiments or opinions, while opinions and sentiments are often exchangeable. We have two major types of documentary information: opinions and factual information. While opinions are known as subjective lex that are used to identify views of people's feelings or sentiments toward characteristics of events, objects, or entities, facts are objective expressions about events, entities, objects, features, and their physiognomies. Sentimental analysis (sentiment classification, opinion mining, polarity classification, sentiment mining, subjectivity analysis, appraisal extraction or review mining) deals with computational processing of subjective, opinions, and sentiments [17]; its plan is to understand the opinion or attitude of users with respect to a certain goal or topic. The defiance of the user could be reflected by her/his evaluation or opinion, her/his effective circumstances (while the recording of opinion, what are feelings of users), or the emotive persistence communication (while reading the opinion of the user, what are the effects situated on the reader).

Furthermore, it ought to be noted that subjective (in context) does not mean something is not true [18]. In the sentimental analysis, cramming the subjective language in the context of a conversation or text is the language used to swift a private state. The rising eminence of sentimental analysis can play an important role in response to both post-factual and datafication, stressing emotion in areas of inquiry previously understood primarily in analytical and rational terms [19]. In [20], the author acknowledged an individual situation as a universal wrap term for assessment, evaluations, opinions, and emotions. There are three critical types of subjective expressions concerning.

Nevertheless circumstances: e.g., "He was boiling with anger," with references to writing or speech: e.g., "The editors of the left-leaning paper attacked the new house speaker" and concerning communicative subjective entities: for instance, "That doctor is a quack," etc.

It was mentioned earlier that the number of online users is in billions, who use the Internet broadly all over the day and read/write online. So, for the decision-making process, day-to-day sentiments available online turn out to be the most substantial issue. Extensive survey research by "Dimensional Research" discusses the percentage of customer trust affected by online reviews for personal endorsements. According to a study conducted in 2011, $74 \%$ of client's sureness is based on online reviews of personal recommendations, according to research in 2012, it was $60 \%$, and according to study in 2013 , it was $57 \%$. But a survey conducted in 2014 states a huge increment with respect to a percentage as it was $94 \%$ [21]. The growth of sentimental analysis from the media industry across the communications and advertising arena has piloted in a new concentration in the feeling quantification, as well as newly oriented radical turmoil and affect that also joins into procedural discussions within the social sciences [22].

A technique that is widely utilized for a variety of applications, ranging from customer service to marketing for online reviews and social media, is known as sentiment analysis. Sentimental analysis has an approach that can be used to construct a lexicon that has information about positivity or negativity of phrases or words. For instance, a blatantly attainable lexical resource of each WordNet is SentiWordNet. Synset is attributed to unfold how negative, positive, and objective terms are [23]. These lexicons can be acquired automatically or compile manually. The lexical 
annotation usually was done manually, and to classify a new batch of phrases or words, large sets of features are used to train classifiers. The critical issue with polarity classification (document classification) is to determining overall characteristics of sentiment for the whole document, whereas actual sentiment can be expressed within one word or sentence. Sentimental analysis has been extensively explored in many text domains, including movie reviews, product reviews, tweets, and so on [24]. In another scenario, the sentiment could be implicitly expressed, so it is tough to classify and detect. However, for classification of sentiment very beneficial information can be obtained through context around these concealed sentiments. Due to division in the field of sentiment analysis, sentiment classification is often based on document level, sentence level, and word level. Another approach for sentiment mining is on the web. The aim of web-based opinion mining is to extract summarized and tracking of various aspects for subjective information [25]. Synopsizing opinion mining defection (also called sentimental analysis) refers to the usage of $\mathrm{CL}$ (computational linguistics), TA (text analysis), and NLP (natural language processing) to extract and identify subjective information in source materials.

In sentiment analysis system, Lexicon plays an important role. In most existing sentiment lexicons, each sentiment phrases or words are given a score or sentiment label. A new unsupervised approach was proposed in [26] for constructing a target-specific sentiment lexicon to extract opinion targets which classify sentiment polarities of opinion pairs accurately. The method achieves both recall in extraction and high accuracy. The framework extracts and integrates different sources of sentiment information.

\section{Methodology}

Previously, sentimental analysis of text or more precisely negative/positive classification depends on using a classifier and a dataset that applies the classifier into sets of two such as negative -ve and positive + ve. During the test phase, an increased number of documents informatively exhibited so that the classification result will be more accurate. Generally, sentiment detection using machine learning techniques is divided into two types: supervised method and unsupervised methods. Supervised methods focus on creating sentiment classification models by labeling the dataset or the document and thus help in making proper decisions, whereas in unsupervised methods, the dataset or the document is not labeled; instead, they rely on the statistical features of the documents. For this purpose, clustering algorithms are used for sentiment analysis in this approach $[1,27,28]$. With the increasing sentiment analysis techniques, the tools, APIs, and libraries used for sentiment analysis have also been growing. Different researchers have developed various platforms for sentiment analysis. Stanford Sentiment Treebank [29], Natural Language Toolkit (NLTK), which also makes it possible to remove stop words [30], Movie Review Data [31], and TextBlob [32] are some sentiment analysis tools and libraries built in python [1]. In sentiment analysis, the lexicon-based approach is also used, which relies on sentiment lexicons having positive, negative, and neutral terms or tones. The sentiment detection based on lexicon-based properties depends upon the co-occurrence of a set of words contained by a sentiment lexicon $[27,33]$.

\subsection{BoW (Bag of Words)}

A model defined in [34] is widely used for classification of supervised text [6], "Document is a nonordered collection of words, word order is expressed even if the grammar is ignored." During the training in this model, the sentiment dictionary is built using BoW to rely on training information and is used to describe the test procedures between positive and negative documents. For example, if you have the following two documents: (1) smoking is very bad to health and (2) smoking should be prohibited in public areas. The dictionary is based on the BoW: dictionary=, 1: "smoking," 2: "is," 3: "very," 4: "bad," 5: "to," 6: "health," 7 : "should," 8: "be," 9:"be," 10: "in," 11:"public,"12, "areas."Therefore, the feature vector for each document is a dictionary built based on " 12 dimensions." As evidenced by the discussion of emotion analysis, the emergence of "word is very informative (as opposed to the word frequency data acquisition)." According to the landscape of the NL, a word can show the author's behavior clearly when there is no word order. For example, under a sentence, when the entire sentence indicates a positive polarity, only words "like" and "not" indicate the sentence polarity.

\subsection{Lexicon base}

The semantic orientation of reviews or words in the text can be calculated using the lexicon-based approach. According to the literature for the lexicon-based approach, dictionaries can be created manually or automatically using different tools or seed words.

For this thesis, the WordNet dictionaries have been used instead of creating dictionaries manually [35]. WordNet is an enormous lexical base database of English that contains four parts of cognitive synonyms, also called synset. These cognitive synonyms (synsets) consist of "nouns, verbs, adjectives, and adverbs." Synsets are connected by the source of lexical base and conceptual-semantic. There are three main reasons for choosing WordNet for this 
project. Coming to the first one other than WordNet, all dictionaries are based on the Aggregated Dataset, which is the (combination of six small sets). It is enormous and almost not usable for Pro Tools or MATLAB for the constructed matrix. Because in other dictionaries, verbs in which nouns have different types, i.e., singular or plural and unlike tenses in documents that increase the size of the dictionaries inadequately, on the other hand, WordNet dictionary uses lemmatized form. The primary purpose of using this feature is that it does not value the structure of the sentence but act upon the cognitive synonyms or synsets. Secondly, because WordNet is a big dictionary, so for "Aggregated Dataset," it is credible to have all the words as a source in contrast with it.

Nevertheless, "for the small size of the given dataset, it was discussed that the corpus-based method is more useful in opinion classification," in terms of the amount of document with a common subject. It would be right to say that the WordNet dictionary is a substantial help for lexical dictionaries solutions. And lastly, it is an available and most popular dictionary. The results from WordNet can be generalized for other datasets as well because the experimentation conducted on it is free from the corpus.

In short, lexicon-based approach was used to make the dictionaries of the data taken from the IMDB database on the movie reviews and divide it into positive and negative words dictionaries, each with 12,500 words using WordNet.

\subsection{POS (part of speech) tags}

POS (part of speech) encryption/encoding, [36] alias "grammar encoding or word-Class," is the same encoding process word text (corpus) belongs to a specific part of the speech, according to its description, as well as the context in relationship with the adjacent and associated paragraphs, words, or phrases. Tagging grammar structures of each word is a good approach to get the precision of the
POS and sense usable models of classifications. Subjective texts usually use simple past verbs rather than past adjectives. Unlike positive sets, negatives have more frequent verbs in the past tense, for the reason that different writers have different ways to express negative emotions.

\subsection{Semantic relationships}

It is a difference between the meanings of the words "associations, which are in between sentences, or between the definitions of sentences (mantic relationship at the level of the phrase or sentence)." Here is an explanation of this relationship. Semantic approach "will depend on different principles, giving the value of the emotion directly" to calculate the similarity of words. This technique is similar to a semantically dense word. "WordNet, for example, gives a different type of semantic relationship between words that are used to calculate the polarity of the" sentences. WordNet can also be used to get a list of words of emotion by repeating the first expending with synonyms and antonyms and by the relative number of synonyms the word positive and negative that determines the polarity of the emotion of the unknown word. Semantic methods are used to find weaknesses in online journals products; both methods [37] can be mixed with statistical methods of performing as work as a job. The weakness Finder uses the plain method to retrieve features and explicit feature groups to identify the comment's word attribute. They used the HowNet-based similarity measure to find common and intermittent overt properties that describe the same side [38]. They used the meaning meta-ODS to group words according to their corresponding sides. In a grouped product, there is a word on the corresponding side by applying a semantic method.

The network mapping can be efficiently described as shown in Fig. 2.
Fig. 2 An MLP multilayered perceptron

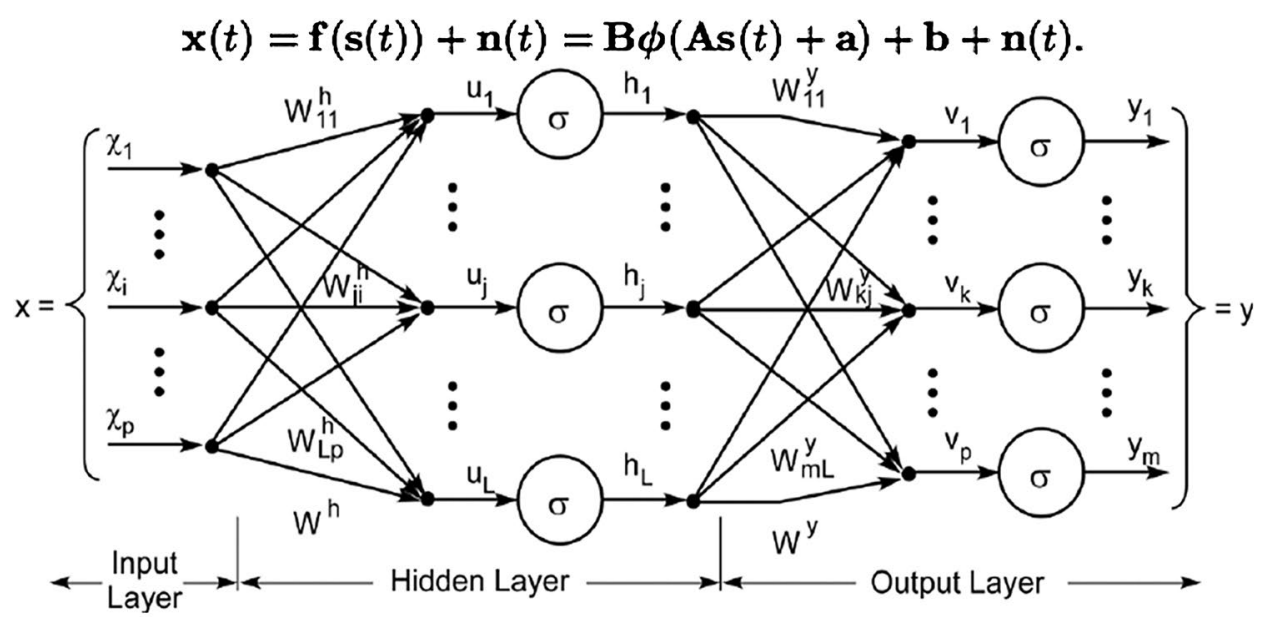

SN Applied Sciences A SPRINGER NATURE journal 


\subsection{Challenges of sentiment analysis}

This section is about the discussion [39] of the problems that sentiment analysis faces. Study in terms of abstraction in the query, a rich set of interconnected sub-issues that make up the ' effective analysis problem. "The assumption is that if we cannot structure the problem," we will probably not understand. The purpose of this definition is to intensify the unstructured nature of the text, both complex and intimidation. "From a practical application standpoint, let's look at the description that professionals should address the problem, the system, and the desired output." See Fig. 3.

\section{State-of-the art implementation}

The ANN (artificial neural network) is a "tool for processing information that is inspired by the way biological nervous systems try to replicate the way the brain processes information. The key feature of this method is the structure

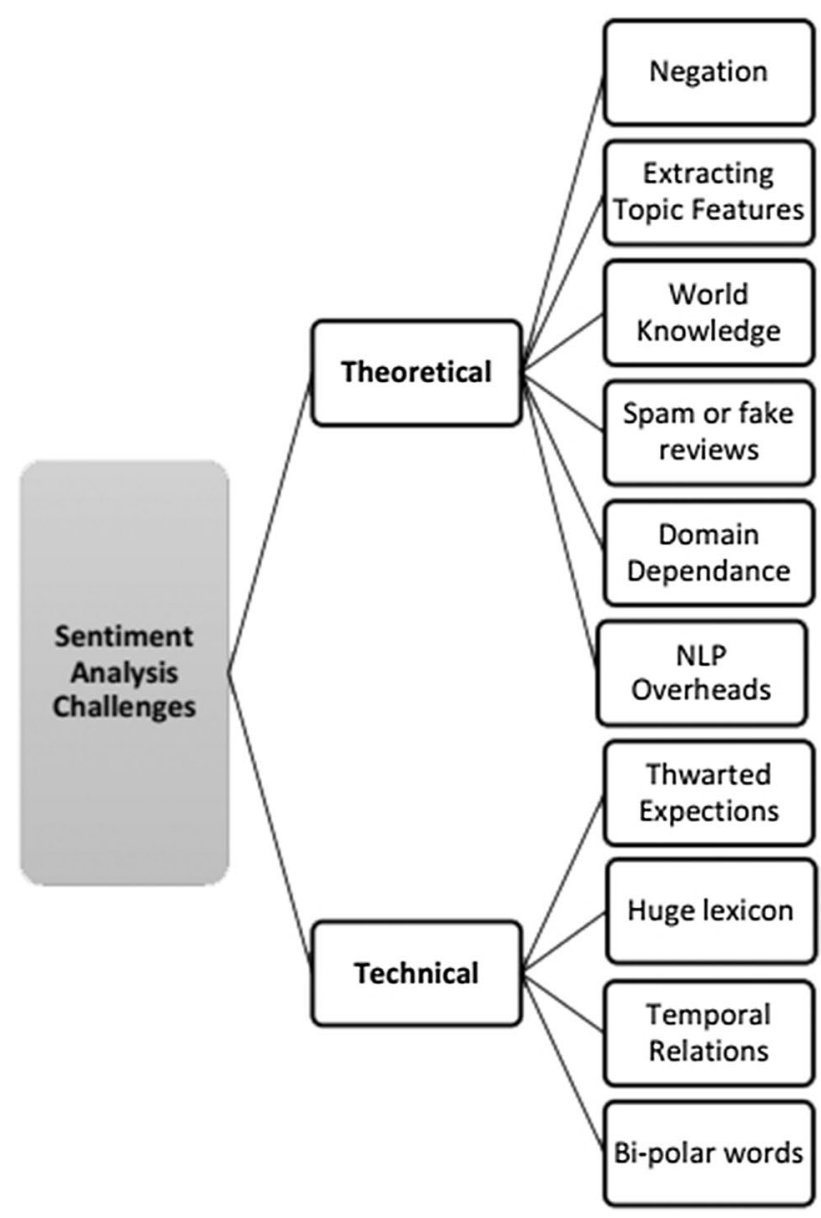

Fig. 3 Challenges of sentimental analysis of the information processing system. It is composed of a large number of highly interconnected processing elements (neurons) working together to solve specific problems." Neural networks, like humans, learn by examples and replication of those examples. A neural network (NN) could be skilled for "specific application, such as pattern recognition or data classification, through a learning process." Learning in nature suggests changes in the synaptic connections existing between the neurons. That also applies to neural networks. For IMDB dataset used for this research, text preprocessing is normalized using the following steps:

All letters are converted to lower or upper case $\rightarrow$ Numbers are removed or converted into words $\rightarrow$ Punctuations, accent marks, and other diacritics are removed $\rightarrow$ White spaces are removed, and abbreviations are expanded $\rightarrow$ Stop words, sparse terms, and particular words are removed $\rightarrow$ Canonicalization of text.

\subsection{Neural networks (NNs)}

The artificial neural network is a developed tool that is used to process information. The motivation behind developing ANN was to imitate "how the human brain processes information," which was stimulated by the biological nervous system. "Information processing system" is the main factor behind the structure of artificial neural networks. Neural networks are a set of huge numbers of strongly connected processing elements called neurons, operating simultaneously to solve a specific problem.

In human beings, neural networks can be studied by the behavior and its repetition. Nowadays, because of machine learning, there are wide research areas that are using neural network application to train for a "specific purpose, i.e., pattern recognition or text classification, using a learning process." The synaptic relations existing among the neurons are also recommended by learning in nature. This relates to neural networks also. "The artificial neural network is a powerful data-driven, self-adaptive, flexible computational tool possessing the capability of capturing nonlinear and complex underlying characteristics of any physical process at a high degree" [34]. Because of this very mature nature, it is often known as a "Black Box." These ANNs perhaps seem to work most of the time independently but still the involvement of humans is required at a certain point. To define the complication or learning rate, it is required to set some "higher level" properties before the model knows about the real parameters. "Hyperparameters" are known as "knobs" of the big machines. Selecting hyperparameter standards corresponds to the prosecution of model selection. Hyperparameters could be "distinct (as in model selection) or continuous." The values of the parameter can be selected manually or adjusted by an

SN Applied Sciences 
algorithm, but the most important thing is to select them carefully.

\subsection{A Simple neuron}

An artificial neuron is basically having a structure that consists of many inputs and only one output. Fundamentally, "the training mode" and "the operation mode" are the two functioning mechanisms that are set in the artificial neuron. The neuron could be trained, i.e., it executes or not in the training mode. And when it approaches the later one (operating mode) for a model in which neuron has been trained through input which is defined, it identifies the pattern of the network, and related output is fired. The firing rules define if it should fire or not if the trained model is not renowned by the input pattern. Below is the figure of a simple neural network. $X$ is defined as an input function, and $W$ is the weight of the inputs.

\subsection{Multilayer perceptron}

Multilayer perceptron is also referred to ANN and which nowadays is the most widely used as a type of neural network. An MLP is made up of a minimum of three layers making it nonlinearly for deep neural networks, which consist of input, hidden, and output layer. A hidden layer has more than one node to construct a deeper architect for neural networks and has a real computational value of MLP. MLP with a single hidden layer is also known as the "vanilla" neural network. A perceptron is a particular neuron model that placed the basis of deeper and more complicated networks. A supervised learning approach can be used for MLP to train and learn about the correlations in between the given input and outputs. In order to reduce the error, we can modify the weights, parameters, and biases during the training process.

MLP is also known as feedforward networks; it is just like a real neural network in human being's information which is continuously moving between input layers to output layers. The basic purpose of it is to create ingenuous replicas of real neural networks so that people may take benefit of solving problems while creating strong data pattern techniques. So, the best practice is to do mapping for NN. Precisely speaking, the ANN can nearly learn "any mapping function" and is accepted to be a "universal approximation algorithm."The analytical competence of neural networks originates from the multilayered arrangement of the networks (Fig. 4).

\subsection{Experimental network}

Network architecture (NA) used in the experiments is explained below. With one output layer and two hidden layers for binary classification, the network employs a "stochastic" gradient lineage for learning and a sigmoid activation function (Fig. 5).
Fig. 4 Diagram of the proposed database

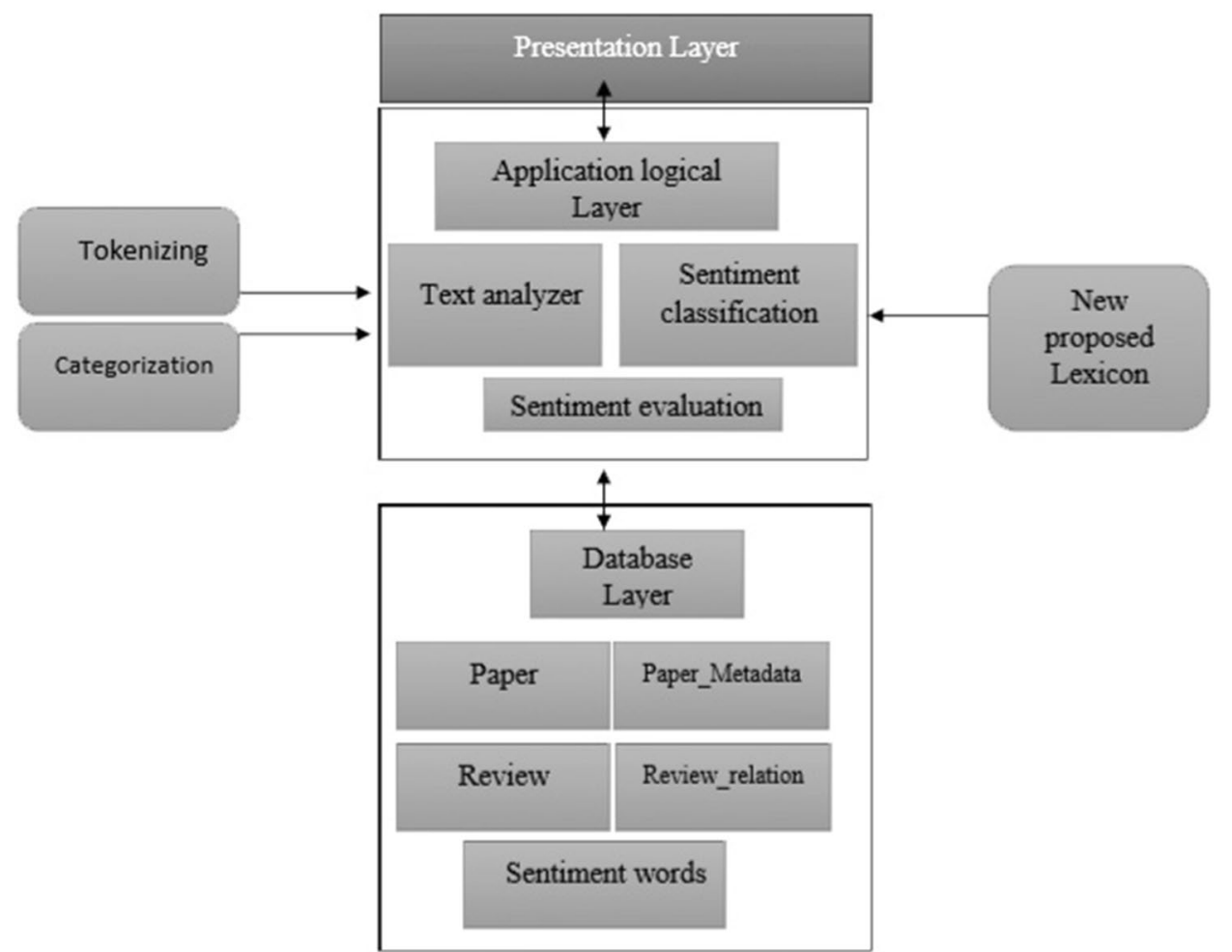

SN Applied Sciences A SPRINGer NATURE journal 
Fig. 5 Architecture used in experiments

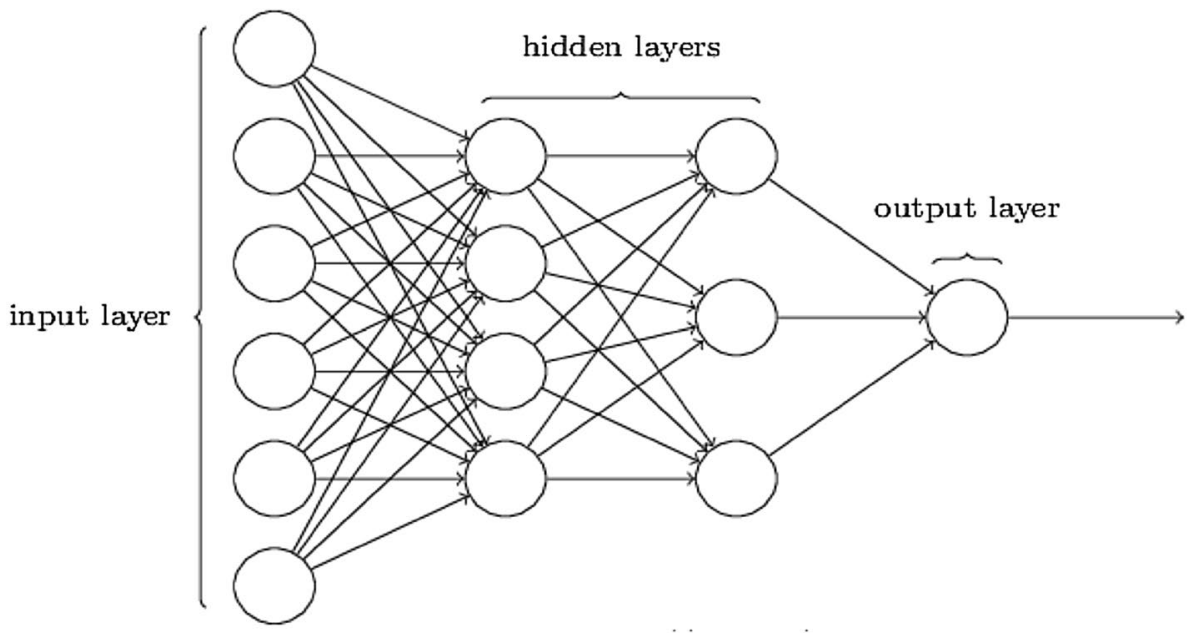

\subsection{Evaluation}

Based on this approach, the proposed sentiment polarity relies on word weight instead of term frequency for each word. Every term has two values and polarity, as proposed in an assumption equation:

$[V w=W p+W n=1]$

where $V w$ is the "word value," Wp goes for "positive word value," and $W n$ stands for "negative word value."The selection between negative polarities and positive polarities is affected by the meanings of a word and each of its polarity. The classification scale of sentiments is between 0 and 1 . In other words, all probable values of each instance cannot exceed one. However, the sentence contains"negative that differs in the word value. If the word is positive, convert to negative polarity and the negative score will be as in the equation":

$[V w=W p-0.4]$

In the case of a negative word, the score will be calculated by the given equation:

$[V w=W n+0.4]$

where 0.4 refers to the assumption level of one to five (1-5) sentiments classification. The proposed technique also "creates ranks by calculating sentiment and measuring domain parameters." This technique is also used for the ranking of the reviews by computing opinions and determining its factors:
$\mathrm{O}(\mathrm{TS})=\sum(\mathrm{O}(\mathrm{SA})+\mathrm{O}(\mathrm{SS})) n k=0$

$\mathrm{O}(\mathrm{TS})$ refers to the overall score for every review. $\mathrm{O}(\mathrm{SA})$ is the overall sentiment score of reports having no. of positives reviews, and O(SS) is the system score (Figs. 6, 7).

T-SNE components of movie embeddings clustered by ratings. It is quite evident that movies which are low rated (blue and green) are concentrated in one portion of the embedding space. Similarly, movies which are highly rated (red and black) are concentrated in another portion of the space. F score is calculated, while weighted averaging is used to evaluate the performance of the classifiers. A good overview of the accuracy provided by the $F$ score incorporates negatives false and positives true. A final accuracy of $91.9 \%$ is managed to achieve by the trained network model (Table 1).

\section{Conclusion}

Unarguably, sentimental analysis techniques are among the utmost significant bases in the decision-making process. A lot of people depend on sentimental analysis for achieving efficient results of services or products. We started with a model that was decent in producing IMBD movie reviews. So, the idea of applying a pre-trained language model to actually outperformed the cutting-edge research in academia as well. It is an undeniable fact that human languages are relatively complex to be understood by the machine, which leads to conditions where a negatively said word has a positive association and vice versa. So, a sentimental analysis of movie reviews was a

Fig. 6 Experimented results

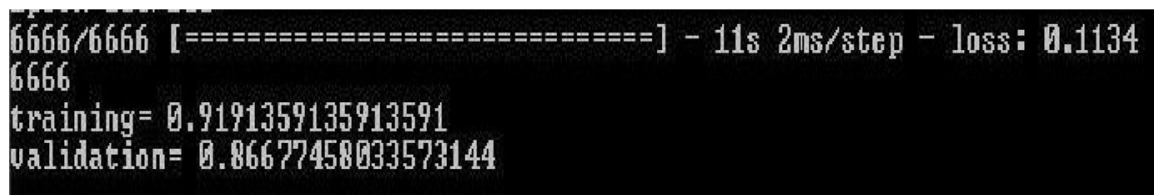


Fig. 7 T-SNE visualization of test data

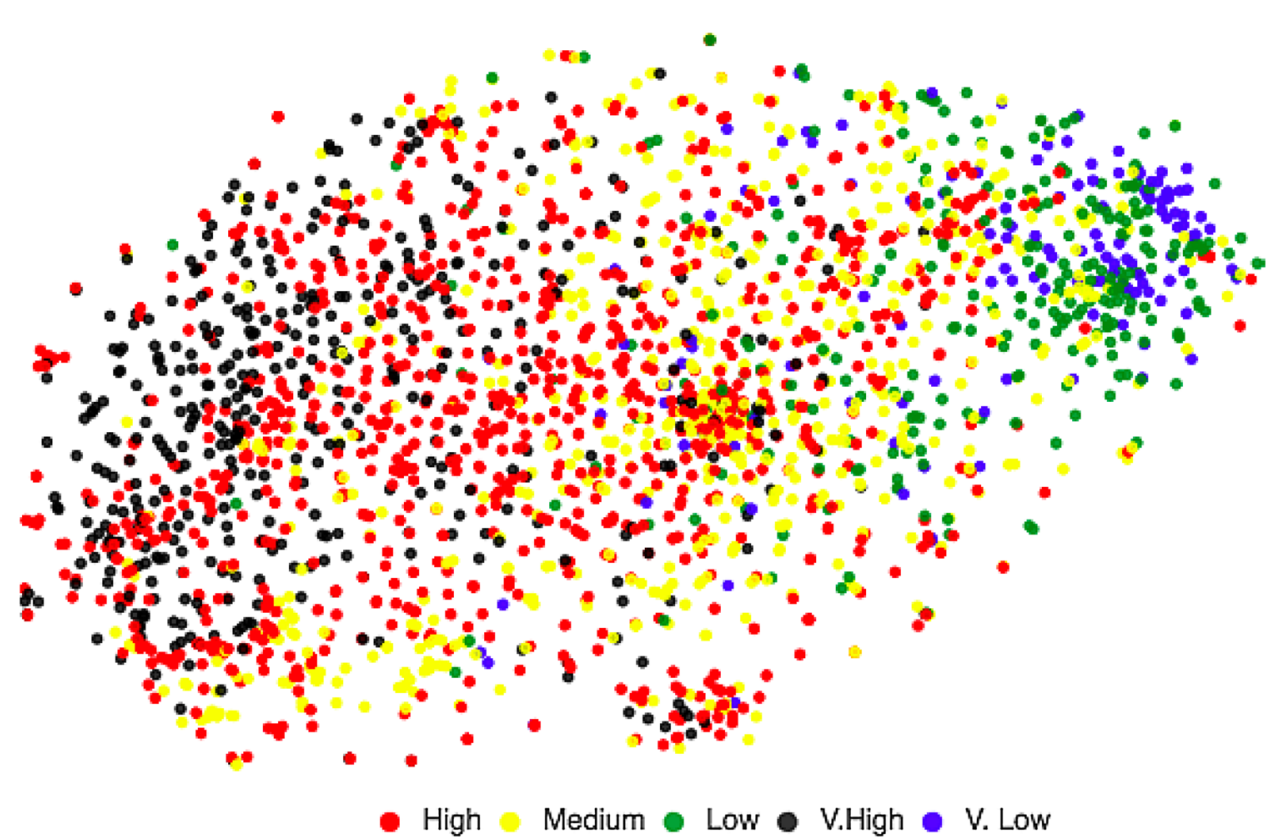

Table 1 Comparison between current and previously achieved accuracy

\begin{tabular}{lll}
\hline Dataset & Scheme & Accuracy \\
\hline IMDB & Lexicon & $91.9 \%$ (current) \\
& & $83.0 \%$ (previous) [1] \\
\hline
\end{tabular}

challenging task. In this study, we used neural networks, which leads us to achieve the task of opinion mining from movie reviews, which was trained on "Movie Review Database by Stanford University" in concurrence with two immense lists of negative and positive words. The trained system accomplished to accomplish an ultimately exceptional final precision. For the movie reviews, a neural network model has been made using the artificial neural network having six layers (four hidden, one input, and one output) having one neuron for binary classification (positive or negative review). The training accuracy of this model has reached $91.9 \%$ and validation accuracy which is $86.67 \%$

\section{Compliance with ethical standards}

Conflict of interest The authors declare that they have no conflict of interest.

\section{References}

1. Giatsoglou M et al (2017) Sentiment analysis leveraging emotions and word embeddings. Exp Syst Appl 69:214-224

2. Kumar HMK, Harish BS, Darshan HK (2018) Sentiment analysis on IMDB movie reviews using hybrid feature extraction method. Int J Interact Multimedia Artif Intell 1-7 (In Press)

3. Hussein DME-DM, A survey on sentiment analysis challenges. J King Saud Univ Eng Sci 30(4): 330-338

4. Khoo CS, Johnkhan SB (2018) Lexicon-based sentiment analysis: Comparative evaluation of six sentiment lexicons. J Inf Sci 44(4):491-511

5. Liu $Y$ et al (2007) ARSA: a sentiment-aware model for predicting sales performance using blogs. In: Proceedings of the 30th annual international ACM SIGIR conference on Research and development in information retrieval, pp 607-614

6. McGlohon M, Glance NS, Reiter Z (2010) Star quality: aggregating reviews to rank products and merchants. In: Fourth international AAAI conference on weblogs and social media

7. O'Connor B et al (2010) From tweets to polls: linking text sentiment to public opinion time series. In: Fourth international AAAI conference on weblogs and social media, vol 11

8. Tumasjan A et al (2010) Predicting elections with twitter: what 140 characters reveal about political sentiment. In Fourth international AAAI conference on weblogs and social media, vol 10

9. Yano T, Smith NA (2010) What's worthy of comment? Content and comment volume in political blogs. In Fourth international AAAI conference on weblogs and social media

10. Olesen Larsen $P$, von Ins $M$ (2010) The Rate of growth in scientific publication and the decline in coverage provided by science citation index. Scientometrics 84:575-603

11. Hudson $S$ et al (2015) The influence of social media interactions on consumer-brand relationships: A three-country study of brand perceptions and marketing behaviors. Int J Res Market 33:27-41

12. Liu B (2012) Sentiment analysis and opinion mining. Synth Lect Hum Lang Technol 5:1-167

13. Ma T (2019) Lexicon extraction from non-parallel data. Google Patents 
14. Cambria E (2016) Affective computing and sentiment analysis. IEEE Intell Syst 31(2):102-107

15. Cruz NP, Taboada M, Mitkov R (2016) A machine-learning approach to negation and speculation detection for sentiment analysis. J Assoc Inf Sci Technol 67(9):2118-2136

16. Banea C, Mihalcea R, Wiebe J (2011) Multilingual sentiment and subjectivity analysis. Multiling Nat Lang Process 6:1-19

17. Agarwal A et al (2011) Sentiment analysis of twitter data. In: Proceedings of the workshop on language in social media (LSM 2011)

18. Benamara F et al (2005) Sentiment analysis: Adjectives and adverbs are better than adjectives alone. In: ICWSM

19. Puschmann C, Powell A (2018) Turning words into consumer preferences: how sentiment analysis is framed in research and the news media. Soc Media Soc 4(3):2056305118797724

20. Liu B (2010) Sentiment analysis and subjectivity Hand of natural language processing. Handb Nat Lang Process 2:627-666

21. Peng L et al (2014) What do seller manipulations of online product reviews mean to consumers?

22. Halford S, Savage M (2017) Speaking sociologically with big data: symphonic social science and the future for big data research. Sociology 51(6):1132-1148

23. Pang $B$, Lee $L$ (2008) Opinion mining and sentiment analysis. J Found Trends Inf Retr 2(1-2):1-135

24. Wang K, Wan X (2018) Sentiment analysis of peer review texts for scholarly papers. In: The 41st International ACM SIGIR conference on research and development in information retrieval. ACM.

25. Jeh G, Widom J (2004) Mining the space of graph properties. In: Proceedings of the tenth ACM SIGKDD international conference on knowledge discovery and data mining. 2004, ACM: Seattle, WA, USA. pp 187-196

26. Wu S et al (2019) Automatic construction of target-specific sentiment lexicon. Exp Syst Appl 116:285-298

27. Al-Asmari S, Dahab M (2017) Sentiment detection, recognition and aspect identification. Int J Comput Appl 177:975-8887

28. Tripathy A, Agrawal A, Rath SK (2016) Classification of sentiment reviews using n-gram machine learning approach. Exp Syst Appl 57(C):117-126
29. Stanford. Sentiment Treebank. [cited 2019; Available from: https ://nlp.stanford.edu/sentiment/treebank.html.

30. Bird S, Klein E, Loper E (2009) Natural language processing with Python: analyzing text with the natural language toolkit. O'Reilly Media Inc, Newton

31. Lee B.P.a.L (2019) Movie review data. [cited 2019; Available from: https://www.cs.cornell.edu/people/pabo/movie-review-data/.

32. Loria S (2019) TextBlob: simplified text processing. [cited 2019; Available from: https://textblob.readthedocs.io/en/dev/autho rs.html.

33. Hamdan $H$, Bellot $P$, Bechet F (2015) Sentiment lexicon-based features for sentiment analysis in short text. Res Comput Sci 90:217-226

34. Jebaseel A, Kirubakaran DE (2012) M-learning sentiment analysis with data mining techniques. Int J Comput Sci Telecommun $3(8): 45-48$

35. Banea C, Mihalcea R, Wiebe J (2008) A bootstrapping method for building subjectivity lexicons for languages with scarce resources. In: LREC

36. Liu BJIIS (2010) Sentiment analysis: a multi-faceted problem. IEEE Intell Syst 25(3):76-80

37. Yumusak $S$ et al (2014) Tagging accuracy analysis on part-ofspeech taggers. J Comput Commun 2(04):157

38. V Presutti et al 2014 Reforgiato Recupero, D., Tordai, A., "Semantic web evaluation challenge. Series title: Communications in computer and information science Springer Berlin

39. Dushyant $M$, Rathod B, Khanna S (2013) A review on emerging trends of web mining and it's applications 1

Publisher's Note Springer Nature remains neutral with regard to jurisdictional claims in published maps and institutional affiliations. 